\title{
Opportunities and Challenges in Using Dynamic Software in Mathematics Education
}

\author{
Soheila B. Shahmohammadi \\ Brock University, Canada
}

\begin{abstract}
The emergence of modern technology, particularly dynamic software, in the teaching and learning of mathematics has raised new challenges for both teachers and students. On the one hand, national organizations such as the National Council of Teachers of Mathematics (NCTM) and the International Society for Technology in Education (ISTE) as well as educational authorities recommend effective integration of technology into mathematics classrooms as the central focus of education, so that students can better understand mathematical ideas and critically solve mathematical problems. On the other hand, some researchers assert that over-use of technology and problems like black-boxing of the central concepts within the use of mathematical software leads to a metacognitive shift that results in students' losing their focus on the mathematical concept to something else. Having considered three popular theories including Information Processing Theory, the Instrumental Theory, and the Semiotic Mediation Theory, and based on the results of a number of relative empirical studies, this paper attempts to illuminate a few functions and limitations of dynamic software, specifically Computer Algebra Systems, Dynamic Geometry Software, and GeoGebra, in the teaching and learning of mathematics.
\end{abstract}

\section{Introduction}

Over the last decades, as a result of the increasing growth of instructional technologies such as computer programs, applications, and software in educational contexts, many studies have been conducted to examine their prevalence, functions, and advantages in school classrooms. The role of technology in educational environments is becoming increasingly prominent because of their potential value as effective learning tools as well as empowering students in our modern societies by developing their computing skill [16]. Findings of a study conducted in Prince Edward Island (Canada) indicate that a wide range of technological tools and activities such as smartboards, computer programs, educational applications, Prezi, flipped classrooms, and teacher blogs are currently used in high schools in Canada. [19]

While teaching and learning of mathematics, among other subject matters, is often considered to be a tough and complicated cognitive endeavor [4], at the same time it has a high practical value in other scientific and technological fields [16]. Much research has been conducted to acknowledge the supportive effects of using instructional tools, such as a graphing calculator, Computer Algebra System (CAS), and Chartworld, in improving teaching and learning of mathematics $[1,4,6]$. Despite the massive affordances of these modern technologies to provide opportunities for visualization, manipulation, and exploration of both geometrical figures and mathematical concepts to students, their usage are not widespread $[1,12,13]$ This paper does not seek the factors affecting infrequent use of dynamic software in mathematics education; instead, it scrutinizes a number of relevant empirical studies to illustrate some possibilities and limitations of these dynamic tools in integrating technology's use by teachers.

\section{Challenges in Using Dynamic Software in Math Education}

The fast growth of modern technologies in mathematics teaching and learning has raised new challenges for teachers since it implies "using new kinds of mathematical tasks, [and] modifying the nature of mathematical activities in classroom based on a set of pedagogical principles" [15, p. 127]. For instance, while Dynamic Software such as Computer Algebra System (CAS), Sketchpad, and GeoGebra, have been recognized as innovative tools with high potential to enhance students' learning of mathematical concepts, a few researchers believe that overuse or "unclear contractual relations about the role of CAS" [9, p.261] lead to students' metacognitive shifts, resulting in a lose of their focus on the original mathematical concept to something else (e.g. specific procedures).

Jankvist and colleagues, who examined three empirical examples of the use of Computer Algebra System (CAS) in a secondary school mathematics class, reported that all examples "lead to didactical problems surrounding the situation and unclear expectations between teacher and students, involving loss of students' mathematical skills and confidence, loss of global mathematical perspective, and the students losing sight of the mathematical objects in question" [9, p. 261]. The authors further argue that 
using some CAS packages in educational settings, while they were initially developed for professional use (e.g. Maple), or easement of students' mathematics work (e.g., Photomath or Cossincalc), might create unforeseen didactical consequences.

In another study, the authors argued that although using Computer Algebra Systems in upper-level secondary schools has high potential for mathematics procedural concepts to be followed, it leads to blackboxing of the central concepts that are the focus of the teaching [8]. This means that although students might hang on to the procedural and pragmatic approaches, at the same time, there is a substantial risk that they do not acquire deep understanding of central concepts and topics.

These critical viewpoints show that there is still need to analyze how technology affects and strengthens students' learning and conceptual understanding of mathematics. Specifically, it seems imperative that teachers need to understand how and through what cognitive process various instrumental tools can help students to enhance their learning of mathematical concepts. This paper attempts to examine how mathematical software act as a cognitive tool in learning mathematical concepts. Having considered the breadth of mathematics software, the focus of this paper will be on how specific dynamic software, namely Computer Algebra Systems (CAS), Dynamic Geometry Software (DGS) such as Sketchpad and Cabri, and Dynamic Mathematical Software (DMS), GeoGebra, affect students' mathematics learning.

To support this aim, first a brief definition of terms is offered, then the theoretical framework of this revision is presented, and finally, some challenging findings of a few empirical studies on using certain dynamic software in teaching and learning mathematics will be discussed.

\section{Dynamic Software in Math Education}

According to the free dictionary, a general definition for mathematical software is "a set of algorithms used in a computer system to solve general mathematical problems" [14]. Over the last decades, various software has been introduced to educational environments. Among them are Computer Algebra Systems (CAS), spreadsheets, and Dynamic Geometry Software (DGS) and all have seen increased use in teaching and learning mathematics [5]. Each has its specific features and is suitable for teaching and learning specific topics in mathematics.

CAS is a type of software for performing algebraic calculations, as well as manipulating and simplifying formulas. It can solve equations, differentiate and integrate functions analytically, and has the potential for numerical calculations and graphing [4]. Popular computer algebra packages that are used in the education domain are Mathcad, Maple, TI-Interactive, and Derive, among others.

DGS is a type of software being used to construct and analyze the tasks and problems in geometry, using the computer mouse and activating a variety of its geometric tools. Two well-known examples of DGS are Geometer's Sketchpad and Cabri Geometry. Although there is a slight difference in their functionality and usage, three essential features are common in DGS and CAS including drag mode, trace of objects, and customizable tools [18]. DGS also can provide a platform to analyze geometric graphs and objects by using a coordinate system as well as focus on function graphs "by creating the locus of a given point whose $\mathrm{y}$-coordinate is calculated using a given expression" [18, p.33].

DMS, GeoGebra is an open source software that combines the possibilities of both DGS and CAS in one single tool for mathematics education. GeoGebra, as a holistic, comprehensive computerbased program, provides a combination of the graphical, numerical and algebraic perspectives of mathematical entities [18]. Moreover, dynamic worksheets are potential interactive Html pages, which can be created with GeoGebra by any Internet browser to provide an e-learning content as well.

\section{Cognitive tools}

Cognitive tools have transformed how mathematics education can be accomplished. Pea [17] defined a cognitive technology as "any medium that helps transcend the limitations of the mind (e.g., attention to goals, short-term memory span) in thinking, learning, and problem-solving activities" [p. 91]. All symbol systems such as writing systems, pictorial media, symbolic computer languages, systems of mathematical notations, and number symbols are considered as cognitive tools [17]. Jonassen [10] defined cognitive tools as generalizable devices that support the learning process and facilitate cognitive processing. Cognitive tools have been considered as computationally based devices that not only complement and extend the mind, but also engage the learners in the generative processing of information by relating new information to prior knowledge. Jonassen [10] pointed out that it is not the technology itself that mediates the process of learning, but rather the mental process of thinking which becomes activated by instructional interventions through technology and other devices, mediates learning. Thereby, learning occurs if the learner efficiently engages with thinking processes in the mind via different types of technologies.

Among all cognitive technologies, written language, number symbols, and systems of mathematical notations have received the most attention. "Each has transformed how mathematics 
can be done and how mathematics education can be accomplished" [17, p.91]. The most predominant feature of all these tools is the externality making of the intermediate products of thinking, which can be reflected upon, analyzed, and interpreted. Vygotsky explained this idea as the "extracortical organizers of thought" since the tools allowed the user to organize thinking outside of the brain [as cited in 16]. Parallel to the idea of 'organizer' role of cognitive tools, pea [17] presented the metaphor of 'amplifier' role of cognitive tools that was introduced by previous scholars. He explained that cognitive technologies, as cultural amplifiers of the intellect, have the capacity to empower human cognition. For instance, the amplifier function of cognitive tools can be considered as the amplification of possibilities, while the user investigates many similar situations cases at a very high speed [4].

Having considered that the potential capacity of cognitive technologies in restructuring thinking process is complex, often indirect, and seldom predictable, Pea [17] asserted the emergent properties of cognitive tools can be discovered only through experimentation. While new goals and ideas for learning activities emerge through experimentation, the adjusted cognitive tools are being continually remade to satisfy the users.

\section{Theoretical Framework}

Cognitive theories provide the opportunity to determine how and through what process and mediation, learning mathematics may occur in our mind. In this section, three popular theories, namely Information Processing Theory, Instrumental Theory, and Semiotic Mediation Theory, which are useful in technology-related researches in the field of mathematics education [5], will be presented briefly. Information Processing Theory simply explains how information is entered, analyzed, stored, and retrieved in humans' minds. Based on this theory, the human brain, like a computer, processes the received information rather than respond the external stimuli [21]. Information Processing Theory explains three main components, namely, sensory memory, working memory, and long-term memory. Sensory memory refers to all the gained experiences through five senses from the environment. Working memory is defined as "a temporary storage system under attentional control that underpins our capacity for complex thought" [2, p. 1]. Willingham defines working memory as "the place in the mind where thinking happens" [21, p. 114]. The salient feature of working memory is its extremely limited capacity. Long-term memory holds information that is much longer lasting and it has a larger capacity than the working memory. In a metaphoric language, longterm memory "is the vast storehouse in which you maintain your factual knowledge of the world" [21, p.14]. Working memory is an assumed place in our brain where learning occurs by combining and processing the information gained from environment-driven and factual knowledge derived from long-term memory to construct new knowledge $[2,21]$.

In the Instrumental Theory, Drijvers and colleagues point out that the distinction between artifact and instrument is the starting point that needs to be considered. An artifact refers to an object that is utilized as a tool, which is a matter of granularity, that is, it depends on the situation of its usage [5]. For instance, in the case of dynamic mathematics software, it depends on if it is considered as a one single artifact, or as a collection of artifacts like the dragging artifact, construction artifact, and so on. Drijvers [5] defined instrument as an artifact that "involves the techniques and mental schemes that the user develops and applies while using the artifact" (p. 108). The central point of The Instrumental Theory is the 'instrumental genesis' which is the process of becoming an artifact to a part or whole of an instrument in the hands of users [5]. Heid [7] explains instrumental genesis as follows:

"It is, of course, not the technology that makes the difference but rather how it is used and by whom. Those who have studied the use of technology in mathematics teaching and learning have noted that technology mediates learning. That is, learning is different in the presence of technology. The representations that students access may conceal or reveal different features of the mathematics, and the procedures students assign to the technology (as opposed to doing them by hand) may affect what students process and learn. Moreover, how a student uses technology is dependent on his or her everchanging relationship to the technology." (p. 348)

Heid explains that a user, as a first step of using a technological tool, may merely encounter the rote application of that particular keystrokes or procedures. However, as the user goes ahead and develops his or her understanding of the capabilities of the technology, that specific tool becomes an instrument that the user can flexibly adjust to specific needs [7].

The Semiotic Mediation Theory, initially introduced by Vygotsky, has been considered as a useful theoretical framework for research concerning integrating technology into mathematics learning [5]. It suggests a strong connection between student learning and tools' usage. According to Drijvers, the mediating potential of artifacts is presented in the dual semantic link that they have with both the meanings that have constructed/emerged during their usage for doing a task and the mathematical meaning that has been aroused by that use, which is identifiable by experts (in our case teachers). In this regard, there might be two considerations about any artifact. One is from the student's point of view, who 
acts with a tool to accomplish the task, and the other one from the social point of view, where experts or mathematics teacher recognize a body of shared meanings [5]. "From a socio-cultural perspective, the tension between these two points of view is the motor of the teaching-learning process centered in the use of an artifact [5, p. 116-117]. However, it is noticeable that despite many theoretical advancements, Drijvers believes that still "the articulation of different theoretical frameworks from different backgrounds is not realized" [5, p. 120], because there are some aspects of the issue that have been remained underexposed, such as the role of the teacher and the language in high-tech learning environments.

\section{Opportunities in Using Dynamic Software in Math Education}

In a longitudinal research study, Drijvers [4] investigated how using Computer Algebra Software can generally enhance the learning of algebra and specifically contribute to high-level understanding among students (grade nine and ten) of the concept of parameter. More than 100 lessons were taught to one hundred and ten students by four trained teachers at a school in Bilthoven, a small town in the province of Utrecht, Netherlands. The results of this research indicated that utilizing CAS supported students remarkably to change their perception of the parameter to a changing quantity through the different representation of algebra and its repetition in different ways. Drijvers [4] reported that over the course of this longitudinal study solving equations with various unknowns by computer algebra system improved students' flexibility with the algebraic symbols, as well as the meaning and the structure of expressions. However, Drijvers conjectured that "integrating CAS into algebra education is better suited for longer periods, so that students would be able to really get used to the tool, or in higher grades, when they have more algebraic experience" [4, p. 295-296]. Moreover, the author emphasized that can emerge serious difficulties for students when underestimating the whole-class discussion, along with the reflection on the consequences of results, and the relation to paper-and-pencil work.

From a theoretical framework perspective, the improvement of mathematical knowledge of students parallel to the computer algebra knowledge development in a reciprocal path in this study can be appropriately justified by the concept of instrumental genesis. As the instrumental genesis emphases on students developing utilization schemes when at first, using a technological tool seems a mechanical rote application of the device, and then progressively by more understanding of tool's potentials and capabilities, students' proficiency in tailoring to their specific needs will increase. It means that by leaving it to the computer algebra system to carry out the procedural activities, students can find the opportunity to concentrate on the concepts involved. In other words, by using computer algebra systems, mathematical entities can be signified and executed by pupils without carrying out the procedures by hand [4]. Additionally, referring to Information Processing Theory, a critical barrier to executing several mental operations simultaneously in the human brain is the limited space of working memory. It means that If you intend to track too many facts or to examine them in too many ways, you lose the main path of what you're doing. One way to improve the working memory capacity is trying to not occupy its limited space by irrelevant or peripheral concepts [21]. In this regard, CAS has the potential to provide more room in working memory for significant operations by executing the marginal operations and leaving students to remain with the tasks in which they are to be performing essential solutions.

In another study, Davoodi [3] conducted a qualitative research study to investigate how students' knowledge and understanding of the concept of the slope of a line and its y-intercept change by using DMS, GeoGebra. First, five students from grade eight and nine from three levels (low, intermediate, and high) were initially interviewed to be determined their understanding as well as their misunderstanding of the concept of the slope and its $y$-intercept in a linear equation to provide appropriate GeoGebra activities for the next step of study. Davoodi selected the students who had already been taught this topic since he aimed to explore in what ways using GeoGebra can change and enhance the students' understanding of this topic. As a second step, two instructional sessions were organized and conducted by the researcher. The first session was held in the group and aimed to introduce the software and its features to students and letting them practice and experience this type of software to have a sense of comfort with it and to identify more details for future structured analysis. The researcher believed that students' interest and familiarity with GeoGebra remarkably influences what the implication of their activities with the software will be. The second session was held individually to explore and clarify the meaning of the slope and y-intercept by interacting with GeoGebra based on the activities that had been carefully designed by the researcher. Then, pupils were again interviewed to respond to the same initial questions, but this time based on their new conceptualization of the intended concepts.

The results of this study that was conducted with Iranian students in London showed that through the designed activities pupils learned a graphical interpretation of the slope and its usage to write an equation for a given line. As there are three ways of 
representations of functions including the tabular, algebraic, and graphical one, Davoodi reported that students' ability to move fluently across the different representations of a function, through appropriate GeoGebra activities, is enhanced remarkably. He asserted that mathematical software without appropriately designed activities and using the contents to exercise and measure the software's power could not help students to understand mathematical concepts [3]. He also emphasized that the process of change in students' knowledge and understanding regarding the slope of a line could be generalized to other mathematical topics if instructors selected suitable computer-based activities.

To investigate whether teaching geometry with Sketchpad, as a dynamic geometry software, affects students' conceptual understanding, inductive reasoning skills, and their motivation towards learning mathematics, Lisa Ames [1] conducted a research in two high school mathematics classes in northern New Jersey with a total of thirty-eight 10th and 11th grade students. Over a six-week intervention period on the unit of properties of quadrilaterals, two classes were compared. The experimental one used Sketchpad and the control group completed the same activities with paper and pencil and other traditional geometric tools, like compasses, protractors, and a ruler. The results of this research showed that the students' inductive reasoning improved by using Sketchpad while there was no significant difference in conceptual knowledge and motivation. However, informal observation in this study indicated that the motivation of students achieving a higher score was greater in the Sketchpad group than those in the control group. Ames [1] suggested that the limited time of the study, the small sample size, lack of randomized sampling, and multiple choice of questions might not capture the students' actual conceptual knowledge of geometry. Not being comfortable or not yet getting used to the geometry in Sketchpad is another reason that Ames reported as the reason for not capturing the conceptual knowledge of quadrilaterals [1].

To add, Falcade et al. [6] conducted a study to examine the potential of a dynamic geometry software, known as Cabri, to provide a proper illustration of both variation and functional dependency by applying Vygotsky's semiotic mediation theory. Having considered "that an organized teaching intervention may foster the move from personal meanings to a mathematical, socially constructed meaning" [6, p.322], researchers designed a teaching experiment to introduce the concept of function to students in four tenth grade classes, of which two were in France and two were in Italy for a period of two months. Based on Vygotsky's perspective, researchers assumed that by using Cabri dragging and trace tool, pupils have the opportunity to comprehend the mathematical meaning of the function. Indeed, a special tool, in this case, Cabri, could act as a semiotic mediator.

Through a broad discussion, Falcade and colleagues explained that Cabri "allows one to feel functional dependency in the basic semantic domain of space and time" [6, p. 320], where the concept of function can be grounded. Having developed the semiotic process, researchers ultimately concluded that Cabri had the essential potentialities for a conceptualizing of function among students. Nevertheless, they believed that teachers had a crucial role "both in the evolution of meanings and in helping students to face the move from describing their activities to the elaboration of a definition" $[6$, p.330].

In a most recent study, Khalil et al.[12] investigated the potential effect of GeoGebra on six aspects of mathematical thinking, namely generalization, abstract thinking, analysis and analytical thinking, problem solving, logical thinking, and representation. This quantitative study was conducted in Pakistan among forty students in grade 12 who were divided into an experimental and a control group through the pair-random sampling process based on their records of grade-11 scores.

Having students in two groups exposed to over 22 lessons in six weeks by the same teacher, a post-test equivalent groups design was conducted to collect data through a test developed by the researchers. By using t-test analysis, the results indicated a significant difference between two groups in five variables including generalization, logical thinking, abstract thinking, analytical thinking, and representation. However, an insignificant difference was found between two groups in the problemsolving variable. Researchers argued that it is necessary to make educational choices based on "objectives, methodologies, role of the teacher and the level of the students while implementing appropriate technology" $[12$, p. 8]. They added that students should be trained in a way that they get the sense of concept with the minimum amount of assistance from a teacher.

Through a critical perspective of CAS-integrated policies into the Danish upper secondary school, Jankvist and Misfeldt [8] discussed that "a strong reliance on CAS provides new and efficient procedural solution strategies to classical problems" (p. 19) that sometimes lead to black-boxing of central concepts of the subject taught in the classroom. Through a theoretical lens, Jankvist and Misfeldt attempted to explain how using CAS in upper secondary school may cause misconceptions or learning difficulties in constructing and developing mathematical concepts. First, they referred to hierarchical stages of developing a new concept, including interiorization, condensation, and 
reification, as introduced by Sfard [as cited in 8 , p.17].

According to this three-stage concept development, while during interiorization, learners become familiar with processes and operations on lower-level mathematical objects and eventually gain skills with these operations, in the stage of condensation learners "squeeze" the sequences of operations and are more able to think of the process as a whole rather than a series of steps. At this stage, learners are able to make comparisons, combine processes, and generalize. Reification occurs when a learner grasps a full-fledged object in itself, and this arises when a new concept is detached from the process that creates it. Second, by referring to Skemp [as cited in 8, p. 17], Jankvist and Misfeldt explained the notion of instrumental understanding and relational understanding. Whereas the former deals with knowing what to do (including procedural knowledge and the knowledge about which procedures to apply), the latter deals with knowing both what to do and why. The authors stated that although the imagination of relational understanding without involving some instrumental understanding is difficult, in a CAS-oriented teaching environment, the reverse direction is possible [8].

Jankvist and Misfeldt discussed that although in a CAS environment, by focusing the students' attention on the intended relevant activity, the lever potential of CAS can save time and enhance the mathematical capacity and the focus of each student in the classroom, at the same time it works by outsourcing (black-boxing) certain mathematical processes that consequently direct attention away from these processes [8]. It means that while students are able to complete CAS-based mathematical activities, they are unable to understand the underlying processes.

\section{Conclusion}

Based on what is presented in this paper, a few important points need to be heeded carefully attention carefully by teachers. The notion of different representational of mathematics knowledge in specific topics such as functions and the slop of the line sets an appropriate stage for mathematical thinking. "Getting involved in recognizing relationships of similarity and different representations forms of the same concept" [3, p.23] is a useful way that leads to a deeper understanding of that concept. This feature is one of the greatest potential strengths of dynamic mathematics software as virtually all the reviewed studies in this paper acknowledged it. Drijvers [4] stated that in a technological environment, while visualization which involves drawing and manipulating graphs, can be done fast, flexibly, and dynamically, the user has the opportunity to link multiple representations of a relation, particularly linking graphical and algebraic representations. In the same way, Karadag and McDougall [11] described the potential of multiple representations of mathematical objects in GeoGebra as students can create a particular mathematical object through one of the representation systems like the algebraic one, then observe its demonstration in other representations like the graphical or verbal one, simultaneously. "Moreover, it is possible to manipulate objects and observe the timely change in their attributes. That is, one can create a slider to control and observe consecutive positions of a point moving" [11, p. 171172].

From a theoretical point of view, based on Information Processing Theory, one way that helps students learn abstract concepts like algebra is exposing them "to many different versions of the abstraction" [24, p. 88]. Therefore, simultaneously presenting the diverse versions of a function allows students to understand the connectivity of its component better. Moreover, Karadag and McDougall [11] stated that "storing some information at a place accessible as needed" will reduce students' cognitive load (p. 171). Thus, it can be concluded that the potential features of multirepresentational as well as dragging and trace of dynamic software, which lead to the visibility of students' action and their feedback, can lead students to deep understanding of mathematics concepts by exposing students to various forms of a concept concurrently. This is in line with Willingham's [23] words that exposing and comparing various examples of an abstract idea force students to think about the deep structure of that abstraction.

However, the students' level of comfort with the cognitive tools before conducting the research is a crucial factor that influences the results of the research. In this regard, Ames [1] strongly recommended that researchers provide a tutorial and adequate hands-on training with Sketchpad before commencing the study. Otherwise, "students' cognitive load might be used to learn the program itself, rather than mathematics" [1, p. 65]. Drijvers [4] also believed that "adequately using a CAS requires insight into notational, syntactic and conceptual matters and is not just a question of 'leaving the work to the computer'. The technical work is related to conceptual understanding." (p. 87)

Moreover, the crucial role of well-designed activities as well as teachers' roles in properly directing students to software use should not be underestimated. Teachers not only play a vital role "in establishing the socio-mathematical norms and the didactical contract" [as cited in 14, p. 241], but they are also the primary source of feedback for students for stimulating their reflection on the work through raising the key issues, making suggestions, and prompting whole-class discussion. These 
strategies may be recommended to teachers as possible remedies to alleviate what Jankvist and Misfeldt [8] called Cas-induced difficulties in learning mathematics.

\section{References}

[1] Ames, L. (2011). The effect of incorporating Geometer's Sketchpad in a high school geometry course to improve conceptual understanding, inductive reasoning, and motivation (Doctoral dissertation). The William Paterson University of New Jersey

[2] Baddeley, A. (2007). Working memory, thought, and action (Vol. 45). OUP Oxford.

[3] Davoodi, K. (2009). Investigating learning mathematics using dynamic software: A case study of understanding the concept of slope (Master's thesis). University of London

[4] Drijvers, P. H. M. (2003). Learning algebra in a computer algebra environment: Design research on the understanding of the concept of parameter (Doctoral dissertation). Utrecht University, Netherlands.

[5] Drijvers, P., Kieran, C., Mariotti, M. A., Ainley, J., Andresen, M., Chan, Y. C., ... \& Meagher, M. (2009). Integrating technology into mathematics education: Theoretical perspectives. In Mathematics education and technology-rethinking the terrain (pp. 89-132). Springer US.

[6] Falcade, R., Laborde, C., \& Mariotti, M. A. (2007). Approaching functions: Cabri tools as instruments of semiotic mediation. Educational Studies in Mathematics, 66(3), 317-333. DOI 10.1007/s10649-006-9072-y

[7] Heid, M. K. (2005). Technology in mathematics education: Tapping into visions of the future. Technology-supported mathematics learning environments, 67, 345 .

[8] Jankvist, U. T., \& Misfeldt, M. (2015). CASinduced difficulties in learning mathematics? For the Learning of Mathematics, 35(1), 15-20.

[9] Jankvist, U. T., Misfeldt, M., \& Marcussen, A. (2016). The didactical contract surrounding CAS when changing teachers in the classroom. Journal of Research in Mathematics Education, 5(3), 263-286.

[10] Jonassen D.H. (1992) What are Cognitive Tools? In: Kommers P.A.M., Jonassen D.H., Mayes J.T., Ferreira A. (eds) Cognitive Tools for Learning.
NATO ASI Series (Series F: Computer and Systems Sciences), vol 81. Springer, Berlin, Heidelberg

[11] Karadag, Z., \& McDougall, D. (2011). GeoGebra as a cognitive tool. Model-Centered Learning, 169-181.

[12] Khalil, M., Sultana, N., \& Khalil, U. (2017). Exploration of Mathematical Thinking and its Development through GeoGebra. Journal of Educational Research, 20(1), 83.

[13] Little, C. (2008). Interactive geometry in the classroom: old barriers and new opportunities. Proceedings of the British Society for Research into Learning Mathematics, 28(2), 49-54.

[14] Mathematical Software. (n.d.) McGraw-Hill Dictionary of Scientific \& Technical Terms, 6E. (2003).

[15] Moreno, M., \& Llinares, S. (2018). Prospective Mathematics Teachers' Perspectives on Technology. In Educating Prospective Secondary Mathematics Teachers (pp. 125-142). Springer, Cham.

[16] Ng'eno, J., Githua, B., \& Changeiywo, J. (2013). Teachers' perceptions of their preparedness to integrate information communication and technology in secondary school mathematics instruction in Rift Valley Region, Kenya. Journal of Education and Practice, 4(12), 2013.

[17] [16] Pea, R. D. (1987). Cognitive technologies for mathematics education. Cognitive science and mathematics education, 89-122.

[18] Preiner, J. (2008). Introducing dynamic mathematics software to mathematics teachers: the case of GeoGebra (Doctoral thesis). University of Salzburg, Austria.

[19] Preston, J. P., Moffatt, L., Wiebe, S., McAuley, A., Campbell, B., \& Gabriel, M. (2015). The use of technology in Prince Edward Island (Canada) high schools: Perceptions of school leaders. Educational Management Administration \& Leadership, 43(6), 989-1005. DOI: $10.1177 / 1741143214535747$

[20] Sfard, A. (1991) - Mathed.net Wiki, http://mathed.net/wiki/Sfard_(1991) (accessed January 14, 2018).

[21] Willingham, D. (2009). Why don't students like school? A cognitive scientist answers questions about hot the mind works and what it means for the classroom. Jossey-Bass, San Francisco, CA. 